\title{
Front Matter: Volume 9050
}

, "Front Matter: Volume 9050," Proc. SPIE 9050, Metrology, Inspection, and Process Control for Microlithography XXVIII, 905001 (13 May 2014); doi: $10.1117 / 12.2052784$

SPIE. Event: SPIE Advanced Lithography, 2014, San Jose, California, United States 


\section{PROCEEDINGS OF SPIE}

\section{Metrology, Inspection, and Process Control for Microlithography XXVIII}

Jason P. Cain

Martha I. Sanchez

Editors

24-27 February 2014

San Jose, California, United States

Sponsored by

SPIE

Cosponsored by

NOVA Ltd. (United States)

Published by

SPIE 
The papers included in this volume were part of the technical conference cited on the cover and title page. Papers were selected and subject to review by the editors and conference program committee. Some conference presentations may not be available for publication. The papers published in these proceedings reflect the work and thoughts of the authors and are published herein as submitted. The publisher is not responsible for the validity of the information or for any outcomes resulting from reliance thereon.

Please use the following format to cite material from this book:

Author(s), "Title of Paper," in Metrology, Inspection, and Process Control for Microlithography XXVIII, edited by Jason P. Cain, Martha I. Sanchez, Proceedings of SPIE Vol. 9050 (SPIE, Bellingham, WA, 2014) Article CID Number.

ISSN: 0277-786X

ISBN: 9780819499738

Published by

SPIE

P.O. Box 10, Bellingham, Washington 98227-0010 USA

Telephone +1 3606763290 (Pacific Time) · Fax +1 3606471445

SPIE.org

Copyright @ 2014, Society of Photo-Optical Instrumentation Engineers.

Copying of material in this book for internal or personal use, or for the internal or personal use of specific clients, beyond the fair use provisions granted by the U.S. Copyright Law is authorized by SPIE subject to payment of copying fees. The Transactional Reporting Service base fee for this volume is $\$ 18.00$ per article (or portion thereof), which should be paid directly to the Copyright Clearance Center (CCC), 222 Rosewood Drive, Danvers, MA 01923. Payment may also be made electronically through CCC Online at copyright.com. Other copying for republication, resale, advertising or promotion, or any form of systematic or multiple reproduction of any material in this book is prohibited except with permission in writing from the publisher. The CCC fee code is 0277-786X/14/\$18.00.

Printed in the United States of America.

Publication of record for individual papers is online in the SPIE Digital Library.

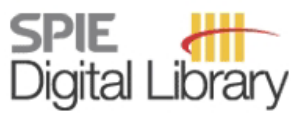

SPIEDigitalLibrary.org

Paper Numbering: Proceedings of SPIE follow an e-First publication model, with papers published first online and then in print and on CD-ROM. Papers are published as they are submitted and meet publication criteria. A unique, consistent, permanent citation identifier (CID) number is assigned to each article at the time of the first publication. Utilization of CIDs allows articles to be fully citable as soon as they are published online, and connects the same identifier to all online, print, and electronic versions of the publication. SPIE uses a six-digit CID article numbering system in which:

- The first four digits correspond to the SPIE volume number.

- The last two digits indicate publication order within the volume using a Base 36 numbering

system employing both numerals and letters. These two-number sets start with 00, 01, 02, 03, 04, $05,06,07,08,09,0 A, 0 B \ldots 0 Z$, followed by 10-1Z, 20-2Z, etc.

The CID Number appears on each page of the manuscript. The complete citation is used on the first page, and an abbreviated version on subsequent pages. Numbers in the index correspond to the last two digits of the six-digit CID Number. 


\section{Contents}

\section{Part One}

xv Conference Committee

HYBRID AND VIRTUAL METROLOGY TECHNIQUES

905004 Optimizing hybrid metrology through a consistent multi-tool parameter set and uncertainty model [9050-3]

R. M. Silver, B. M. Barnes, N. F. Zhang, H. Zhou, A. Vladár, J. Villarrubia, J. Kline, D. Sunday, National Institute of Standards and Technology (United States); A. Vaid,

GLOBALFOUNDRIES Inc. (United States)

905005 Leveraging data analytics, patterning simulations and metrology models to enhance CD metrology accuracy for advanced IC nodes [9050-4]

N. Rana, Y. Zhang, T. Kagalwala, L. Hu, T. Bailey, IBM Semiconductor Research and Development Ctr. (United States)

905006 New techniques in large scale metrology toolset data mining to accelerate integrated chip technology development and increase manufacturing efficiencies [9050-5] E. Solecky, N. Rana, A. Minns, C. Gustafson, P. Lindo, IBM Systems and Technology Group (United States); R. Cornell, P. Llanos, Applied Materials, Inc. (United States)

905008 CDSEM AFM hybrid metrology for the characterization of gate-all-around silicon nano wires [9050-7]

S. Levi, I. Schwarzband, Y. Weinberg, Applied Materials, Inc. (Israel); R. Cornell, Applied Materials, Inc. (United States); O. Adan, Applied Materials, Inc. (Israel); G. M. Cohen,

L. Gignac, S. Bangsaruntip, IBM Thomas J. Watson Research Ctr. (United States); S. Hand,

J. Osborne, A. Feinstein, Bruker Nano Inc. (United States)

905009 Hybrid metrology universal engine: co-optimization [9050-8]

A. Vaid, C. Osorio, J. Tsai, GLOBALFOUNDRIES Inc. (United States); C. Bozdog,

M. Sendelbach, Nova Measuring Instruments Inc. (United States); E. Grubner, R. Koret,

S. Wolfling, Nova Measuring Instruments Ltd. (Israel)

\section{METROLOGY OF 3D STRUCTURES}

9050 OA 10nm three-dimensional CD-SEM metrology [9050-9]

A. E. Vladár, J. S. Villarrubia, National Institute of Standards and Technology (United States);

J. Chawla, Intel Corp. (United States); B. Ming, J. R. Kline, National Institute of Standards and Technology (United States); S. List, Intel Corp. (United States); M. T. Postek, National Institute of Standards and Technology (United States)

9050 OB Optical technologies for TSV inspection [9050-71]

A. A. Aiyer, N. Maltsev, J. Ryu, Frontier Semiconductor, Inc. (United States) 
9050 0C Addressing FinfET metrology challenges in 1X node using tilt-beam CD-SEM [9050-11] X. Zhang, GLOBALFOUNDRIES Inc. (United States); H. Zhou, Z. Ge, Applied Materials, Inc. (United States); A. Vaid, D. Konduparthi, C. Osorio, GLOBALFOUNDRIES Inc. (United States); S. Ventola, Applied Materials, Inc. (United States); R. Meir, O. Shoval, R. Kris, O. Adan, M. Bar-Zvi, Applied Materials, Inc. (Israel)

9050 OD Novel three dimensional (3D) CD-SEM profile measurements [9050-12] W. Ito, Advantest Corp. (Japan); B. Bunday, SEMATECH Inc. (United States); S. Harada, Advantest Corp. (Japan); A. Cordes, SEMATECH Inc. (United States); T. Murakawa, Advantest Corp. (Japan); A. Arceo, SEMATECH Inc. (United States); M. Yoshikawa, T. Hara, T. Arai, S. Shida, M. Yamagata, J. Matsumoto, T. Nakamura, Advantest Corp. (Japan)

9050 OF Metrology of white light interferometer for TSV processing [9050-14]

P. Timoney, Y.-U. Ko, D. Fisher, C. K. LU, Y. Ramnath, A. Vaid, S. Thangaraju, D. Smith, H. Kamineni, D. Zhang, W. Kim, R. Alapati, GLOBALFOUNDRIES Inc. (United States); J. Peak, H. Amin, H. Edmunson, J. Race, B. Peterson, T. Johnson, Nanometrics Inc. (United States)

SEM SIMULATION AND EMULATION I: JOINT SESSION WITH CONFERENCES 9050 AND 9051

9050 0G Influence of metrology error in measurement of line edge roughness power spectral density [9050-15]

B. D. Bunday, SEMATECH Inc. (United States); C. A. Mack, Lithoguru.com (United States)

$9050 \mathrm{Ol} \quad$ New integrated Monte Carlo code for the simulation of high-resolution scanning electron microscopy images for metrology in microlithography [9050-17]

E. Ilgüsatiroglu, A. Yu. Illarionov, M. Ciappa, ETH Zürich (Switzerland); P. Pfäffli, L. Bomholt, Synopsys Switzerland, LLC (Switzerland)

$9050 \mathrm{OJ}$ Correction of EB-induced shrinkage in contour measurements [9050-18] T. Ohashi, S. Hotta, A. Yamaguchi, J. Tanaka, Hitachi, Ltd. (Japan); H. Kawada, Hitachi High-Technologies Corp. (Japan)

9050 OK Dependence of secondary-electron yield on aspect ratio of several trench patterns [9050-19]

D. Bizen, Y. Sohda, Hitachi, Ltd. (Japan); H. Kazumi, Hitachi High-Technologies Corp. (Japan)

SEM SIMULATION AND EMULATION II: JOINT SESSION WITH CONFERENCES 9050 AND 9051

9050 OL Determination of line edge roughness in low dose top-down scanning electron microscopy images (2014 Karel Urbanek Best Student Paper Award) [9050-20]

T. Verduin, P. Kruit, C. W. Hagen, Technische Univ. Delft (Netherlands)

9050 OM Cross-sectional profile prediction from top-view SEM images based on root-cause decomposition of line-edge roughness [9050-21]

H. Fukuda, Hitachi High-Technologies Corporation (Japan) 
METROLOGY AND INSPECTION FOR DIRECTED SELF-ASSEMBLY: JOINT SESSION WITH CONFERENCES 9049 AND 9050

9050 ON Metrology for directed self-assembly block lithography using optical scatterometry [9050-22]

D. Dixit, College of Nanoscale Science \& Engineering (United States); V. Kamineni, R. Farrell, E. Hosler, M. Preil, GLOBALFOUNDRIES Inc. (United States); J. Race, B. Peterson, Nanometrics Inc. (United States); A. C. Diebold, College of Nanoscale Science \& Engineering (United States)

$905000 \quad$ Novel metrology methods for fast 3D characterization of directed self-assembly (DSA) patterns for high volume manufacturing [9050-23]

C. Sarma, B. Bunday, A. Cepler, SEMATECH Inc. (United States); T. Dziura, KLA-Tencor Corp. (United States); J. Kim, G. Lin, J. Yin, AZ Electronic Materials USA Corp. (United States)

\section{METROLOGY FOR PROCESS CONTROL}

9050 OP Estimating pattern sensitivity to the printing process for varying dose/focus conditions for RET development in the sub-22nm era [9050-24]

B. Seguin, H. Saab, M. Gabrani, IBM Research - Zürich (Switzerland); V. Estellers, Ecole Polytechnique Fédérale de Lausanne (Switzerland)

$90500 Q \quad$ Lithography run-to-run control in high mix manufacturing environment with a dynamic state estimation approach [9050-25]

M. E. Yelverton, G. K. Agrawal, GLOBALFOUNDRIES Inc. (United States)

9050 OR Improvement of inter-field CDU by using on-product focus control [9050-26]

K. D. Park, T. Park, J. H. Hwang, J. P. Choi, Y. S. Kang, SAMSUNG Electronics Co., Ltd. (Korea, Republic of)

9050 OS Improving on-product performance at litho using integrated diffraction-based metrology and computationally designed device-like targets fit for advanced technologies (incl. FinFET) [9050-27]

K.-H. Chen, G. T. Huang, K. S. Chen, C. W. Hsieh, Y. C. Chen, C. M. Ke, T. S. Gau, Y. C. Ku, Taiwan Semiconductor Manufacturing Co. Ltd. (Taiwan); K. Bhattacharyya, J. Huang, A. den Boef, M. v. d. Schaar, M. Maassen, R. Plug, Y. Zhang, S. Meyer, M. van Veen, C. de Ruiter, J. Wu, H. XU, T. Chow, C. Chen, E. Verhoeven, P. Li, P. Hinnen, G. Storms, K. Pao, G. Zhang, C. Fouquet, ASML Netherlands B.V. (Netherlands); T. Mori, Tokyo Electron Ltd. (Japan)

SEM, AFM, AND SPM

9050 OT CD-SEM metrology for sub-10nm width features [9050-28]

B. Bunday, A. Cepler, A. Cordes, A. Arceo, SEMATECH Inc. (United States)

9050 OU Improving SEM image quality using pixel super resolution technique [9050-29]

M. Lee, J. Cantone, J. Xu, L. Sun, R. Kim, GLOBALFOUNDRIES Inc. (United States) 
9050 OV Contour-based metrology for complex 2D shaped patterns printed by multiple-patterning process [9050-30]

D. Fuchimoto, T. Ishimoto, H. Shindo, H. Sugahara, Hitachi High-Technologies Corp.

(Japan); Y. Toyoda, Hitachi, Ltd. (Japan); J. Mailfert, IMEC (Belgium) and Katholieke Univ. Leuven (Belgium); P. De Bisschop, IMEC (Belgium)

9050 OW Parallel SPM cantilever arrays for large area surface metrology and lithography [9050-31] T. Gotszalk, Wrocław Univ. of Technology (Poland); T. Ivanov, I. W. Rangelow, Technische Univ. Ilmenau (Germany)

\section{X-RAY SCATTERING METHODS}

905012 Nanometrology on gratings with GISAXS: FEM reconstruction and fourier analysis [9050-36] V. Soltwisch, J. Wernecke, A. Haase, Physikalisch-Technische Bundesanstalt (Germany); J. Probst, M. Schoengen, Helmholtz-Zentrum Berlin (Germany); M. Krumrey, F. Scholze, Physikalisch-Technische Bundesanstalt (Germany)

OVERLAY MEASUREMENT AND CONTROL: JOINT SESSION WITH CONFERENCES 9050 AND 9052

905013 Monitoring process-induced overlay errors through high-resolution wafer geometry measurements [9050-37]

K. T. Turner, Univ. of Pennsylvania (United States); P. Vukkadala, S. Veeraraghavan, J. K. Sinha, KLA-Tencor Corp. (United States)

905014 Investigation on reticle heating effect induced overlay error [9050-38] M. Lim, G. Kim, S. Kim, B. Lee, S. Kim, C. Lim, M. Kim, S. Park, SK Hynix, Inc. (Korea, Republic of)

905015 Compensating process non-uniformity to improve wafer overlay by RegC [9050-39]

P. Leray, S. Cheng, IMEC (Belgium); A. Cohen, E. Graitzer, V. Dmitriev, S. Rehtan,

N. Wertsman, Carl Zeiss SMS Ltd. (Israel)

INSPECTION

905016 Optical volumetric inspection of sub-20nm patterned defects with wafer noise [9050-40] B. M. Barnes, F. Goasmat, M. Y. Sohn, H. Zhou, A. E. Vladár, R. M. Silver, National Institute of Standards and Technology (United States); A. Arceo, SEMATECH Inc. (United States)

905017 9nm node wafer defect inspection using visible light [9050-41]

R. Zhou, C. Edwards, G. Popescu, L. L. Goddard, Univ. of Illinois at Urbana-Champaign (United States)

905018 Highly effective and accurate weak point monitoring method for advanced design rule (1x nm) devices [9050-42]

J. Ahn, S. Seong, M. Yoon, I.-S. Park, H. Kim, D. Ihm, S. Chin, SAMSUNG Electronics Co., Ltd. (Korea, Republic of); G. Sivaraman, M. Li, R. Babulnath, C. H. Lee, S. Kurada, C. Brown,

R. Galani, J. Kim, KLA-Tencor Corp. (United States) 
905019 Real-time inspection system utilizing scatterometry pupil data [9050-43]

J. Y. Baek, Univ. of California, Berkeley (United States); P. Leray, A.-L. Charley, IMEC

(Belgium); C. J. Spanos, Univ. of California, Berkeley (United States)

9050 1A New inspection technology for observing nanometer size defects using expansion soft template [9050-44]

S. Morita, R. Yoshikawa, T. Hirano, T. Higashiki, Toshiba Corp. (Japan)

9050 1B Parallel, miniaturized scanning probe microscope for defect inspection and review [9050-45]

H. Sadeghian, TNO (Netherlands) and Technische Univ. Delft (Netherlands);

T. C. van den Dool, W. E. Crowcombe, R. W. Herfst, J. Winters, G. F. I. J. Kramer, N. B. Koster, TNO (Netherlands)

$90501 \mathrm{C}$ Computational techniques for determining printability of real defects in EUV mask pilot line [9050-47]

P. Morgan, D. Rost, D. Price, MP Mask Technology Ctr., LLC (United States); Y. Li, D. Peng,

D. Chen, P. Hu, N. Corcoran, D. Son, D. Yonenaga, V. Tolani, Luminescent Technologies

(United States)

9050 1D Quantitative tabletop coherent diffraction imaging microscope for EUV lithography mask inspection [9050-46]

B. Zhang, D. E. Adams, M. D. Seaberg, D. F. Gardner, E. R. Shanblatt, H. Kapteyn,

M. Murnane, Univ. of Colorado at Boulder (United States) and JILA (United States)

\section{SCATTEROMETRY AND OPTICAL METHODS}

$9050 \mathrm{lE} \quad$ Novel in-line metrology methods for Fin pitch walking monitoring in $14 \mathrm{~nm}$ node and beyond [9050-119]

R. Chao, K. Kohli, Y. Zhang, A. Madan, G. R. Muthinti, A. J. Hong, D. Conklin, J. Holt,

T. C. Bailey, IBM Semiconductor Research and Development Ctr. (United States)

$9050 \mathrm{IF}$ Weak measurements applied to process monitoring using focused beam scatterometry [9050-49]

T. G. Brown, M. A. Alonso, A. Vella, M. J. Theisen, S. T. Head, Institute of Optics, Univ. of Rochester (United States)

9050 1G Enhanced optical CD metrology by hybridization and azimuthal scatterometry [9050-50]

S. Zangooie, HGST, Inc. (United States); J. Li, K. Boinapally, Nanometrics Inc. (United States);

P. Wilkens, A. Ver, B. Khamsepour, H. Schroder, J. Piggot, HGST, Inc. (United States);

S. Yedur, Z. Liu, J. Hu, Nanometrics Inc. (United States)

$9050 \mathrm{1H} \quad$ High speed optical metrology solution for after etch process monitoring and control [9050-51]

A.-L. Charley, P. Leray, W. Pypen, S. Cheng, IMEC (Belgium); A. Verma, C. Mattheus, B. Wisse, H. Cramer, H. Niesing, S. Kruijswijk, ASML Netherlands B.V. (Netherlands)

905011 Visualization of Si surface and interface quality by non-contact optical characterization techniques [9050-52]

W. S. Yoo, K. Kang, T. Ishigaki, T. Ueda, WaferMasters, Inc. (United States) 
9050 i I Integrated ADI optical metrology solution for lithography process control of CD and OV [9050-107]

M. Strobl, W. Tsai, A. Lan, T. Chen, W. Hsu, H. Chen, Inotera Taiwan (Taiwan); F. Liang,

A. Wang, P. Hung, D. Huang, E. Chiu, P. Yu, ASML Taiwan (Taiwan); Y. Song, S. Yuan,

R. Dirks, N. Wright, M. Ponomarenko, H. Cramer, B. Wisse, V. Couraudon, B. Rajasekharan,

R. Plug, S. Kruijswijk, H. Niesing, ASML Netherlands B.V. (Netherlands)

\section{REFERENCE METROLOGY, ACCURACY, STANDARDS}

$90501 \mathrm{~K}$ Sidewall roughness and line profile measurement of photoresist and finfET features by cross-section STEM and TEM image for reference metrology [9050-53]

K. Takamasu, H. Okitou, S. Takahashi, The Univ. of Tokyo (Japan); O. Inove, H. Kawada, Hitachi High-Technologies Corp. (Japan); V. Kamineni, A. Paul, A. F. Bello,

GLOBALFOUNDRIES Inc. (United States)

$90501 \mathrm{~L} \quad$ Verification metrology system by using inline reference metrology [9050-54]

H. Abe, Y. Ishibashi, C. Ida, A. Hamaguchi, T. Ikeda, Y. Yamazaki, Toshiba Corp. (Japan)

$90501 \mathrm{M}$ Impact of shrinking measurement error budgets on qualification metrology sampling and cost [9050-55]

M. Sendelbach, Nova Measuring Instruments Inc. (United States); N. Sarig, Nova Measuring Instruments Ltd. (Israel); K. Wakamoto, Nova Measuring Instruments K.K. (Japan); H. K. Kim, Nova Measuring Instruments (Korea, Republic of); P. Isbester, Nova Measuring Instruments Inc. (United States); M. Asano, K. Matsuki, Toshiba Corp. (Japan); A. Vaid, C. Osorio, GLOBALFOUNDRIES Inc. (United States); C. Archie, Consultant (United States)

\section{Part Two}

\section{OVERLAY}

9050 iN Innovative fast technique for overlay accuracy estimation using archer self calibration (ASC) [9050-103]

S. C. C. Hsu, C. Chen, C. C. Yu, Y. C. Pai, United Microelectronics Corp. (Taiwan); E. Amit, KLA-Tencor Israel (Israel); L. Yap, KLA-Tencor Corp. (United States); T. Itzkovich, KLA-Tencor Israel (Israel); D. Tien, KLA-Tencor Corp. (United States); E. Huang, K. T. L. Kuo, KLA-Tencor Taiwan (Taiwan); N. Amir, KLA-Tencor Israel (Israel)

905010 Real cell overlay measurement through design based metrology [9050-58] G. Yoo, J. Kim, C. Park, T. Lee, S. Ji, G. Jo, H. Yang, D. Yim, SK Hynix, Inc. (Korea, Republic of); M. Yamamoto, K. Maruyama, B. Park, NGR Inc. (Japan)

9050 IP Integrated production overlay field-by-field control for leading edge technology nodes [9050-59]

W. J. Chung, J. Tristan, K. Gutjahr, L. Subramany, C. Li, Y. Sun, M. Yelverton, Y. K. Kim, J. S. Kim, GLOBALFOUNDRIES Inc. (United States); C.-C. K. Huang, W. Pierson, R. Karur-Shanmugam, B. Riggs, S. Jug, J. C. Robinson, L. Yap, V. Ramanathan, KLA-Tencor Corp. (United States) 
$90501 Q \quad$ Mask contribution to intra-field wafer overlay [9050-60]

W. Chou, H.-M. Chang, C. Y. Chen, United Microelectronics Corp. (Taiwan); M. Wagner, KLA-Tencor Israel (Israel); K.-D. Roeth, S. Czerkas, M. Ferber, KLA-Tencor GmbH (Germany); M. Daneshpanah, KLA-Tencor Corp. (United States); F. Laske, KLA Tencor GmbH (Germany); R. Chiang, KLA-Tencor Taiwan (Taiwan); S. Klein, KLA-Tencor Corp. (United States)

\section{LATE BREAKING NEWS}

9050 IR Innovative techniques for improving overlay accuracy by using DCM (device correlated metrology) targets as reference [9050-104]

W. J. Tzai, S. C. C. Hsu, H. Chen, C. Chen, Y. C. Pai, C.-C. Yu, C. C. Lin, United Microelectronics Corp. (Taiwan); T. Itzkovich, KLA-Tencor Israel (Israel); L. Yap, KLA-Tencor Corp. (United States); E. Amit, KLA-Tencor Israel (Israel); D. Tien, KLA-Tencor Corp. (United States); E. Huang, K. T. L. Kuo, KLA-Tencor Taiwan (Taiwan); N. Amir, KLA-Tencor Israel (Israel)

9050 is Overlay improvements using a real time machine learning algorithm [9050-105] E. Schmitt-Weaver, M. Kubis, W. Henke, D. Slotboom, T. Hoogenboom, J. Mulkens, M. Coogans, P. ten Berge, D. Verkleij, F. van de Mast, ASML Netherlands B.V. (Netherlands)

9050 it Advanced CD-SEM metrology for pattern roughness and local placement of lamellar DSA [9050-123]

T. Kato, A. Sugiyama, K. Ueda, Hitachi High-Technologies Corp. (Japan); H. Yoshida, Hitachi, Ltd. (Japan); S. Miyazaki, AZ Electronic Materials Japan K.K. (Japan); T. Tsutsumi, AZ Electronic Materials Japan Corp. (Japan); J. Kim, Y. Cao, G. Lin, AZ Electronic Materials USA Corp. (United States)

\section{POSTER SESSION}

$90501 \mathrm{U}$ Evaluation of lens heating effect in high transmission NTD processes at the $20 \mathrm{~nm}$ technology node [9050-61]

B. Jeon, S. Lee, L. Subramany, C. Li, S. Pal, S. Meyers, S. Mehta, Y. Wei, D. R. Cho, GLOBALFOUNDRIES Inc. (United States)

9050 IV Automatically high accurate and efficient photomask defects management solution for advanced lithography manufacture [9050-62]

J. Zhu, L. Chen, L. Ma, D. Li, W. Jiang, L. Pan, H. Shen, H. Jia, Shanghai Huali Microelectronics Corp. (China); C. Hsiang, Anchor Semiconductor, Inc. (United States); G. Cheng, L. Ling, S. Chen, J. Wang, W. Liao, G. Zhang, Anchor Semiconductor, Inc. (China)

9050 IW Design of the phase-shifting algorithm for flatness measurement of a mask blank glass [9050-63]

Y. Kim, The Univ. of Tokyo (Japan); K. Hibino, National Institute of Advanced Industrial Science and Technology (Japan); N. Sugita, M. Mitsuishi, The Univ. of Tokyo (Japan) 
$90501 \mathrm{X}$ Precise CD-SEM metrology of resist patterns at around $20 \mathrm{~nm}$ for 0.33 NA EUV lithography [9050-64]

N. Okai, Hitachi America, Ltd. (United States); E. Lavigne, IBM Semiconductor Research and Development Ctr. (United States); K. Hitomi, Hitachi America, Ltd. (United States); S. Halle, IBM Semiconductor Research and Development Ctr. (United States); S. Hotta, Hitachi, Ltd. (Japan); S. Koshihara, Hitachi High-Technologies Corp. (Japan); K. Petrillo, IBM Semiconductor Research and Development Ctr. (United States); A. Yamaguchi, J. Tanaka, Hitachi, Ltd. (Japan); T. Bailey, IBM Semiconductor Research and Development Ctr. (United States)

$90501 Y \quad$ Lithography focus/exposure control and corrections to improve CDU at post etch step [9050-65]

Y. K. Kim, M. Yelverton, J. Tristan, J. Lee, K. Gutjahr, C.-H. Hsu, H. Wei, L. Wang, C. Li,

L. Subramany, W. J. Chung, J. S. Kim, GLOBALFOUNDRIES Inc. (United States);

V. Ramanathan, L. Yap, J. Gao, R. Karur-Shanmugam, A. Golotsvan, P. Herrera, K. Huang,

B. Pierson, KLA-Tencor Corp. (United States)

$90501 \mathrm{Z}$ The metal ions from track filter and its impact to product yield in IC manufacturing [9050-66]

T.-C. Kuo, United Microelectronics Corp. (Taiwan)

905021 In cleanroom, sub-ppb real-time monitoring of volatile organic compounds using protontransfer reaction/time of flight/mass spectrometry [9050-68]

N. Hayeck, LCE, CNRS, Aix-Marseille Univ. (France) and TERA Environnement (France);

P. Maillot, T. Vitrani, N. Pic, STMicroelectronics (France); H. Wortham, S. Gligorovski,

B. Temime-Roussel, A. Mizzi, LCE, CNRS, Aix-Marseille Univ. (France); I. Poulet, TERA

Environnement (France)

905024 Investigation of a methodology for in-film defects detection on film coated blank wafers [9050-73]

A. Kiyotomi, A. Davendorffer, S. Shimura, S. Miyazaki, Tokyo Electron Kyushu Ltd. (Japan); T. Miyagi, S. Ota, K. Haneda, O. Baris, J. Wei, KLA-Tencor Corp. (United States)

905026 Across wafer CD uniformity optimization by wafer film scheme at double patterning lithography process [9050-75]

H.-C. Lin, Y.-L. Li, S.-C. Wang, C.-H. Liu, Z.-S. Wang, J.-Y. Hsuh, Powerchip Technology Corp. (Taiwan)

905027 Defect analysis methodology for contact hole grapho epitaxy DSA [9050-76]

R. Harukawa, M. Aoki, A. Cross, V. Nagaswami, KLA-Tencor Corp. (United States);

S. Kawakami, T. Yamauchi, T. Tomita, Tokyo Electron Kyushu Ltd. (Japan); S. Nagahara, Tokyo Electron Ltd. (Japan); M. Muramatsu, T. Kitano, Tokyo Electron Kyushu Ltd. (Japan)

905028 Defect analysis and alignment quantification of line arrays prepared by directed selfassembly of a block copolymer [9050-77]

C. Simão, Catalan Institute of Nanoscience and Nanotechnology (Spain); D. Tuchapsky, Univ. College Cork (Ireland); W. Khunsin, Catalan Institute of Nanoscience and Nanotechnology (Spain) and Hokkaido Univ. (Japan); A. Amann, Univ. College Cork (Ireland); M. A. Morris, Tyndall National Institute (Ireland) and Univ. College Cork (Ireland); C. M. Sotomayor Torres, Catalan Institute of Nanoscience and Nanotechnology (Spain) and Catalan Institute of Research and Advanced Studies (Spain) 
905029 New robust edge detection methodology for qualifying DSA characteristics by using CD SEM [9050-79]

S. Yamaguchi, K. Ueda, T. Kato, N. Hasegawa, Hitachi High-Technologies Corp. (Japan);

T. Yamauchi, S. Kawakami, M. Muramatsu, Tokyo Electron Kyushu Ltd. (Japan);

S. Nagahara, Tokyo Electron Ltd. (Japan); T. Kitano, Tokyo Electron Kyushu Ltd. (Japan)

$90502 \mathrm{~B} \quad$ An analytical method for the measurement of trace level acidic and basic AMC using liquid-free sample traps [9050-81]

T. M. Moulton, E. C. Zaloga, K. M. Chase, J. M. Lobert, Entegris, Inc. (United States)

$90502 \mathrm{D}$ A method for the combined measurement of volatile and condensable organic AMC in semiconductor applications [9050-83]

C. M. Miller, E. C. Zaloga, J. M. Lobert, Entegris, Inc. (United States)

$90502 \mathrm{E} \quad$ Handling, clamping, and alignment evaluation for multi-beam technology on Matrix 1.1 platform [9050-85]

L. Lattard, J. Pradelles, CEA-LETI (France); N. Vergeer, E. Slot, MAPPER Lithography (Netherlands); L. Pain, CEA-LETI (France); E. de Jong, MAPPER Lithography (Netherlands); G. Torriani, Dainippon Screen Deutschland GmbH (Germany); C. Pieczulewski, SOKUDO Co., Ltd. (Japan)

$90502 \mathrm{~F} \quad$ Focus control budget analysis for critical layers of flash devices [9050-86] J. H. Jang, T. Park, K. D. Park, J. H. Hwang, J. P. Choi, Y. S. Kang, SAMSUNG Electronics Co., Ltd. (Korea, Republic of)

$90502 \mathrm{G} \quad$ Macroscopic exploration and visual quality inspection of thin film deposit [9050-87] S.-F. Désage, G. Pitard, H. Favrelière, M. Pillet, Univ. de Savoie (France); O. Dellea, P. Fugier, P. Coronel, E. Ollier, CEA-Liten (France)

$90502 \mathrm{H} \quad$ Wafer surface pre-treatment study for micro bubble free of lithography process [9050-88] X. Yang, X. Zhu, S. Cai, Semiconductor Manufacturing International Corp. (China)

9050 2J Run time scanner data analysis for HVM lithography process monitoring and stability control [9050-90] W. J. Chung, Y. K. Kim, J. Tristan, C. Li, L. Subramany, J. S. Kim, GLOBALFOUNDRIES Inc. (United States); B. Riggs, V. Ramanathan, R. Karur-Shanmugam, G. Hoo, J. Gao, A. Golotsvan, K. Huang, B. Pierson, KLA-Tencor Corp. (United States)

9050 2K Isolation mounts scatterometry with RCWA and PML [9050-91] H. Shirasaki, Tamagawa Univ. (Japan)

$90502 \mathrm{~L}$ Data fusion for CD metrology: heterogeneous hybridization of scatterometry, CDSEM, and AFM data [9050-92]

J. Hazart, N. Chesneau, G. Evin, A. Largent, A. Derville, POLLEN Technology SAS (France) and CEA-LETI (France); R. Thérèse, S. Bos, CEA-LETI (France); R. Bouyssou, C. Dezauzier, STMicroelectronics (France); J. Foucher, POLLEN Technology SAS (France) and CEA-LETI-Minatec (France)

$90502 \mathrm{M} \quad$ Accurate contour extraction from mask SEM image [9050-93]

I. Santo, A. Higuchi, M. Anazawa, H. Bandoh, Holon Corp. (Japan) 
$90502 \mathrm{~N}$ Improvement of CD-SEM mark position measurement accuracy [9050-94]

K. Kasa, K. Fukuhara, Toshiba Corp. (Japan)

905020 Mean offset optimization for multi-patterning overlay using Monte Carlo simulation method [9050-95]

W. Wang, L. Cui, L. Sun, R.-H. Kim, GLOBALFOUNDRIES Inc. (United States)

$90502 \mathrm{2}$ 20nm MOL overlay case study [9050-97]

L. Subramany, M. Hsieh, C. Li, H. P. Koh, D. Cho, GLOBALFOUNDRIES Inc. (United States); A. Golotsvan, V. Ramanathan, R. Karur Shanmugam, L. Yap, KLA-Tencor Corp. (United States)

$90502 R \quad$ Metrology of advanced N14 process pattern split at lithography [9050-98]

W.-J. Tzai, C.-C. Lin, C.-H. Chen, C.-C. Yu, United Microelectronics Corp. (Taiwan);

W.-Y. Chu, S. Yoo, C.-J. Huang, C.-Y. Cheng, H.-F. Su, KLA-Tencor Corp. (United States)

905025 Development of UV inspection system on the defect of electrode for $5 \mu \mathrm{m}$ level multilayer pattern [9050-99]

K. Namgung, J. Woo, S. Lim, S. Lee, J. Lee, S. Lim, Myongji Univ. (Korea, Republic of); J. Lim, B. Kim, Auros Technology, Inc. (Korea, Republic of); J. Kim, Myongji Univ. (Korea, Republic of)

$90502 \mathrm{~T} \quad$ Scatterometry performance improvement by parameter and spectrum feed-forward [9050-100]

J. Li, Nanometrics Inc. (United States); S. Zangooie, HGST, Inc. (United States); K. Boinapally,

X. Zou, J. Hu, Z. Liu, S. Yedur, Nanometrics Inc. (United States); P. Wilkens, A. Ver, R. Cohen,

B. Khamsepour, HGST, Inc. (United States)

$90502 \mathrm{U} \quad$ Spectroscopic critical dimension technology (SCD) for directed self-assembly [9050-101] S. Nishibe, T. Dziura, V. Nagaswami, KLA-Tencor Corp. (United States); R. Gronheid, IMEC (Belgium)

9050 2V SEM-contour shape analysis based on circuit structure for advanced systematic defect inspection [9050-102]

Y. Toyoda, Hitachi, Ltd. (Japan); H. Shindo, Y. Hojo, D. Fuchimoto, Hitachi High-

Technologies Corp. (Japan)

9050 2W Real-time focus and overlay measurement by the use of fluorescent markers [9050-106] D. Maas, E. van Zwet, TNO (Netherlands)

$90502 \mathrm{X} \quad$ High-precision self-tool CD matching with focus-target assist pattern by computational ways [9050-108]

S.-M. Kim, H.-C. Kim, J.-W. Lee, Y.-S. Kim, Y.-H. Kim, S.-K. Won, S. Kim, SAMSUNG Electronics Co., Ltd. (Korea, Republic of); K.-Y. Park, C.-H. Ryu, Q.-T. Fan, K.-H. Baik, ASML Brion (Korea, Republic of)

$90502 Y \quad$ In-line focus monitoring and fast determination of best focus using scatterometry [9050-109] S. T. Ha, B. Eynon, M. Wynia, J. Schmidt, SAMSUNG Austin Semiconductor LLC (United States); C. Sparka, A. Mani, R. Volkovich, S. Yoon, D. Tien, J. Robinson, S. Ramamurthi, KLATencor Corp. (United States) 
$90502 Z$ Comparative defect classifications and analysis of Lasertec's M1350 and M7360 [9050-110] M. Godwin, D. Balachandran, SEMATECH Inc. (United States); T. Tamura, Lasertec Corp. (Japan); A. Jia, Lasertec USA Inc. (United States)

905030 Tracking defectivity of EUV and SADP processing using bright-field inspection [9050-11 1] N. Alexis, C. Bencher, Y. Chen, H. Dai, K. Dotan, D. Huang, A. Nalven, C. Ngai, Applied Materials, Inc. (United States); G. Santoro, Applied Materials, Inc. (Belgium);

B. Vijayaraghavan, P. Xie, J. Xue, Applied Materials, Inc. (United States)

905031 Improved reticle requalification accuracy and efficiency via simulation-powered automated defect classification [9050-1 12]

S. Paracha, B. Eynon, B. F. Noyes III, A. Nhiev, Samsung Austin Semiconductor (United States); A. Vacca, P. Fiekowsky, D. Fiekowsky, AVI Photomask (United States); Y. M. Ham, D. Uzzel, M. Green, S. MacDonald, J. Morgan, Photronics, Inc. (United States)

905032 Direct-scatterometry-enabled PEC model calibration with two-dimensional layouts [9050-113]

Y.-Y. Yang, H.-P. Lee, C.-H. Liu, H.-Y. Yu, K.-Y. Tsai, J.-H. Li, National Taiwan Univ. (Taiwan)

905033 Implementation of background scattering variance reduction on the rapid nano particle scanner [9050-114]

P. van der Walle, S. Hannemann, D. van Eijk, W. Mulckhuyse, J. C. J. van der Donck, TNO (Netherlands)

905034 The measurement uncertainty of CD measurement in the optical measurement technology using Fourier image [9050-115]

K. Nagashima, H. Abe, M. Oote, Y. Yamazaki, Toshiba Corp. (Japan)

905035 Process control using set-membership vector-form affine projection adaptive filtering scheme [9050-1 16]

C.-C. Hu, K.-H. Chen, National Chung Cheng Univ. (Taiwan)

905036 Phase shift focus monitor for OAl and high NA immersion scanners [9050-117]

H. M. Kuo, R. C. Peng, H. H. Liu, Taiwan Semiconductor Manufacturing Co. Ltd. (Taiwan)

905037 CD uniformity optimization at volume ramp up stage for new product introduction [9050-118]

J.-S. Kim, W.-K. Ma, Y.-S. Kim, M.-S. Kim, W.-T. Kwon, S.-K. Park, SK Hynix, Inc. (Korea, Republic of); P. Nikolsky, M. Otter, M. E. Marun, R. Anunciado, K.-T. Sun, G. Storms, E. van West, ASML Netherlands B.V. (Netherlands)

905038 Plasma etched surface scanning inspection recipe creation based on bidirectional reflectance distribution function and polystyrene latex spheres [9050-120]

T. Saldana, S. McGarvey, S. Ayres, Hitachi High Technologies America (United States)

905039 The effect of individually-induced processes on image-based overlay and diffractionbased overlay [9050-57]

S. Oh, J. Lee, S. Lee, C. Hwang, G. Choi, H.-K. Kang, E. Jung, SAMSUNG Electronics Co., Ltd. (Korea, Republic of)

Author Index 


\title{
Conference Committee
}

\author{
Symposium Chair
}

Harry J. Levinson, GLOBALFOUNDRIES Inc. (United States)

Symposium Co-chair

Mircea V. Dusa, ASML US, Inc. (United States)

Conference Chair

Jason P. Cain, Advanced Micro Devices, Inc. (United States)

Conference Co-chair

Martha I. Sanchez, IBM Research - Almaden (United States)

Conference Program Committee

Ofer Adan, Applied Materials (Israel)

John A. Allgair, GLOBALFOUNDRIES Inc. (United States)

Masafumi Asano, Toshiba Corporation (Japan)

Benjamin D. Bunday, SEMATECH Inc. (United States)

Alek C. Chen, ASML Taiwan Ltd. (Taiwan)

Shaunee Y. Cheng, IMEC (Belgium)

Timothy F. Crimmins, Intel Corporation (United States)

Daniel J. C. Herr, The University of North Carolina at Greensboro

(United States)

Chih-Ming Ke, Taiwan Semiconductor Manufacturing Co. Ltd. (Taiwan)

Shunsuke Koshihara, Hitachi High-Technologies Corporation (Japan)

Yi-Sha Ku, Industrial Technology Research Institute (Taiwan)

Byoung-Ho Lee, Ultratech (United States)

Christopher J. Raymond, Nanometrics Inc. (United States)

John C. Robinson, KLA-Tencor Corporation (United States)

Matthew J. Sendelbach, Nova Measuring Instruments Inc. (United States)

Richard Silver, National Institute of Standards and Technology

(United States)

Eric Solecky, IBM Corporation (United States)

Costas J. Spanos, University of California, Berkeley (United States)

Alexander Starikov, I\&I Consulting (United States)

Vladimir A. Ukraintsev, Nanometrology International, Inc. (United States)

Alok Vaid, GLOBALFOUNDRIES Inc. (United States) 
Session Chairs

1 Keynote Session

Martha I. Sanchez, IBM Almaden Research Center (United States)

2 Hybrid and Virtual Metrology Techniques

John C. Robinson, KLA-Tencor Corporation (United States)

Matthew J. Sendelbach, Nova Measuring Instruments Inc. (United States)

3 Metrology of 3D Structures

Masafumi Asano, Toshiba Corporation (Japan)

Alok Vaid, GLOBALFOUNDRIES Inc. (United States)

4 SEM Simulation and Emulation I: Joint Session with Conferences 9050 and 9051

Shunsuke Koshihara, Hitachi High-Technologies Corporation (Japan)

Thomas I. Wallow, ASML US, Inc. (United States)

5 SEM Simulation and Emulation II: Joint Session with Conferences 9050 and 9051

Benjamin D. Bunday, SEMATECH Inc. (United States)

Clifford L. Henderson, Georgia Institute of Technology (United States)

6 Metrology and Inspection for Directed Self-Assembly: Joint Session with Conferences 9049 and 9050

Martha I. Sanchez, IBM Research - Almaden (United States)

Daniel J. C. Herr, The University of North Carolina at Greensboro (United States)

$7 \quad$ Metrology for Process Control

Costas J. Spanos, University of California, Berkeley (United States)

Alexander Starikov, I\&I Consulting (United States)

8 SEM, AFM, and SPM

Masafumi Asano, Toshiba Corporation (Japan)

Shunsuke Koshihara, Hitachi High-Technologies Corporation (Japan)

$9 \quad$ X-Ray Scattering Methods

John A. Allgair, GLOBALFOUNDRIES Inc. (United States)

Richard M. Silver, National Institute of Standards and Technology

(United States)

10 Overlay Measurement and Control: Joint Session with Conferences 9050 and 9052

Alexander Starikov, I\&I Consulting (United States)

Pary Baluswamy, Micron Technology, Inc. (United States) 
11 Inspection

Timothy F. Crimmins, Intel Corporation (United States)

Chih-Ming Ke, Taiwan Semiconductor Manufacturing Co. Ltd. (Taiwan)

12 Scatterometry and Optical Methods

Matthew J. Sendelbach, Nova Measuring Instruments Inc. (United States) Eric Solecky, IBM Corporation (United States)

13 Reference Metrology, Accuracy, Standards

Benjamin D. Bunday, SEMATECH Inc. (United States)

Alexander Starikov, I\&I Consulting (United States)

14 Overlay

Chih-Ming Ke, Taiwan Semiconductor Manufacturing Co. Ltd. (Taiwan) John C. Robinson, KLA-Tencor Corporation (United States)

15 Late Breaking News

Jason P. Cain, Advanced Micro Devices, Inc. (United States)

Ofer Adan, Applied Materials (Israel) 